\title{
Der Fluch der Schönheit
}

— Vor Jahren wurde ich im ärztlichen Notdienst von einer besorgten Mutter zu ihrer 30-jährigen Tochter gerufen, die so depressiv war, dass sie seit Tagen ihr Bett nicht mehr verlassen hatte. Die junge Frau hatte verheulte Augen und sprach nur sehr zaghaft und stockend. Trotz ihrem verheulten Gesicht sah man auf den ersten Blick, dass es sich um eine gut aussehende junge Frau mit schönem Gesicht und schlankem wohlgeformten Körper handelte. Genau dieses Aussehen aber war ihr Problem, wie sie mir mitteilte.

Ich mutmaßte zuerst, dass die Patientin eine Dysmorphophobie haben könnte, dann erklärte sie aber Folgendes: Als sie 20
Jahre alt war, sei sie wunderschön gewesen. Viele Männer hätten sie umschwärmt, darunter auch Millionäre und betuchte Playboys. Sie sei auf den wunderbarsten und teuersten Partys auf Ibiza, Mallorca, in Nizza und Monte Carlo dabei gewesen, habe in den besten Hotels gewohnt und in den besten Restaurants gegessen.

Oft sei sie Freitag nachmittags in einen Privatjet nach Ibiza gestiegen und sei amSonntagabend mit dem Taxi vom Flugplatz nach Hause gebracht worden.

Alles auf Einladung. So ging das mehrere Jahre. Mit 27/28 Jahren seien die Einladungen aber erheblich weniger geworden und seit zwei Jahren würden sie vollständig ausbleiben. Sie sei vom Jetset „abgeschrieben".

Sie zeigte mir auch Fotos von damals und ich musste eingestehen: Wenngleich sie auch heute noch sehr apart war, früher war sie wunderschön. Ganz vereinzelt hatte sie jetzt auch schon ein graues Haar.

Mir war klar, dass eine nachhaltige Therapie auf die Schnelle nicht möglich war, bot ihr deshalb eine Krankenhauseinweisung als Möglichkeit an, was die junge Patientin aber rigoros ablehnte. Ich bot ihr daraufhin einen Psychotherapieplatz bei mir an und ließ meine Visitenkarte zurück. Ich habe aber nie mehr von ihr gehört.

DR. MED. RAINER HAKIMI, STUTTGART .

\section{Bissiges Kleinkind}

- Lautes Kindergeschrei ertönte im Anmeldebereich der Poliklinik für Berufskrankheiten Berlin-Lichtenberg. Ein etwa drei Jahre alter Junge wurde gebracht, der die Verbindungsleitung zwischen Steckdose und abgeschalteter Tischlampe in den Mund genommen und solange darauf herumgekaut hatte, bis es zu einem Lichtbogenüberschlag innerhalb der Mundhöhle mit Verbrennungen an der Zunge gekommen war. Offensichtlich hatte das Kind zuerst die Isolierung des Nullleiters durchgebissen, sodass bei nachfolgender Freilegung der Phase ein Lichtbogen entstand, ohne dass eine Durchströmung des Körpers erfolgte. Der Kurzschluss führte zur Abschaltung des gesamten Stromkreises durch Herausspringen der Sicherung.

Das EKG zeigte einen normalen Erregungsablauf. Bei primärem Bisskontakt mit der Phase wäre es zu einer Ganzkörperdurchströmung Mundhöhle-Füße mit Einbeziehung des Herzens in den Unfallstromkreis gekommen. Das Kind wurde unverzüglich mit Begleitung in die Charité verlegt, über den weiteren Verlauf wurde uns nichts bekannt.

DR. MED. E. TÖPPICH, ULLERSDORF •

Hatte ich jetzt etwa eine neue Indikati-

- Morgens um 6:10 rief mich in meinem Nachtdienst ein besorgter Sohn an und erklärte mir, seine Mutter habe einen Blutdruck von $211 / . .$. . Auf meine Frage, ob sie denn ihre Blutdruckmedikamente einnehme, antwortete er: "Ja, jede Menge“. Ich bat ihn, nachzusehen, ob ein Notfall-Nitrospray dabei wäre. Aus dem Hintergrund hörte ich seine Frau rufen: „Ja, hier ist so ein Spray!
Prima, dachte ich, und instruierte ihn, der Mutter davon zwei Hübe unter die Zunge zu geben, in einer Viertel Stunde noch einmal den Blutdruck zu messen und mir dann den Wert durchzugeben.

Um 6:40 Uhr klingelte das Telefon erneut und er Sohn berichtete mir, der Wert läge nun bei 183/.... Das Spray habe übrigens "Spiriva Respimat" geheißen. on entdeckt oder hatte hier die Natur ihre Finger im Spiel? Jedenfalls sagte ich ihm, er solle der Mutter gleich die morgendlichen RR-Medikamente geben und dann den Hausarzt konsultieren, sobald der wieder erreichbar wäre.

DR. Med. Claudia Mentel, KIRCHHeim - 